\title{
BETTER GROWTH IS PROTECTIVE AGAINST THE DEVELOPMENT OF RETINOPATHY OF PREMATURITY IN EXTREMELY PRETERM INFANTS
}

\section{LUND}

UNIVERSITY

Anna-My Lund ${ }^{a}$, Chatarina Löfqvist ${ }^{b}$, Pia Lundgren ${ }^{b}$, Anna-Lena Hård ${ }^{b}$, Ann Hellström ${ }^{b}$, Ingrid Hansen-Pupp ${ }^{a}$ aLund University, Skåne University Hospital, Department of Clinical Sciences, Paediatrics, Lund, Sweden

bSection for Opthalmology, Department of Clinical Neuoroscience, Institute of Neuroscience and Physiology, Sahlgrenska Academy, University of Gothenburg, Gothenburg, Sweden

\section{AIM \\ To investigate the relationship between postnatal growth during hospital stay and development of retinopathy of prematurity (ROP).}

\section{BACKGROUND}

The risk for a preterm infant to develop ROP is, among other factors, related to:

- Low gestational age and low birth weight z-score

- Postnatal weight gain

- Low total energy intake in the early postnatal period

\section{STUDY DESIGN}

Daily nutritional- and breast milk intakes during the first 28 postnatal days, in addition to growth measurements (weight, length and head circumference) and development of neonatal morbidities was prospectively collected from birth until discharge in a Swedish cohort of 90 extremely preterm infants.

\section{RESULTS}

Neonatal characteristics are presented in Table 1. Infants without $\mathrm{ROP}$ received a higher mean enteral energy $(\mathrm{kcal} / \mathrm{kg} / \mathrm{d})$ intake during postnatal days 2-28 than infants with $\operatorname{ROP}(p=0.026)$.

Table 1. Neonatal characteristics, mean nutritional intakes postnatal days 2 to 28 and presence of ROP. Data is presented as either median (min-max) or $n(\%)$

\begin{tabular}{|c|c|c|c|}
\hline & Any ROP ( $n$ 61) & No ROP (n 17) & $P$ value \\
\hline Sex (female) & $31(50.8 \%)$ & $4(23.5 \%)$ & 0.045 \\
\hline Gestational age (weeks) & $25.3(22.9-27.9)$ & $27.0(22.7-27.9)$ & $<0.001$ \\
\hline Birth weight (g) & $725(415-1250)$ & $1020(635-1260)$ & $<0.001$ \\
\hline Birth weight z-score & $-0.69(-5.24-0.43)$ & $-0.08(-2.23-1.28)$ & 0.003 \\
\hline SGA & $10(16.4 \%)$ & $1(5.9 \%)$ & ns \\
\hline EUGR & $14(24.1 \%)^{\circ}$ & $2(14.3 \%)^{b}$ & ns \\
\hline Total energy intake $(\mathrm{kcal} / \mathrm{kg} / \mathrm{d})$ & $111(93-149)$ & $123(92-140)$ & ns \\
\hline Enteral energy intake $(\mathrm{kcal} / \mathrm{kg} / \mathrm{d})$ & $83(4-136)$ & $109(26-134)$ & 0.026 \\
\hline Total protein intake $(\mathrm{g} / \mathrm{kg} /$ day $)$ & $3.6(2.7-5.0)$ & $3.8(2.9-4.4)$ & ns \\
\hline Enteral protein intake $(\mathrm{g} / \mathrm{kg} /$ day) & $2.5(0.1-4.5)$ & $3.4(0.7-4.3)$ & $\mathrm{ns}(0.052)$ \\
\hline First day full enteral nutrition (days) & $15(8-63)^{c}$ & $11(8-31)^{d}$ & 0.042 \\
\hline \multicolumn{4}{|c|}{ 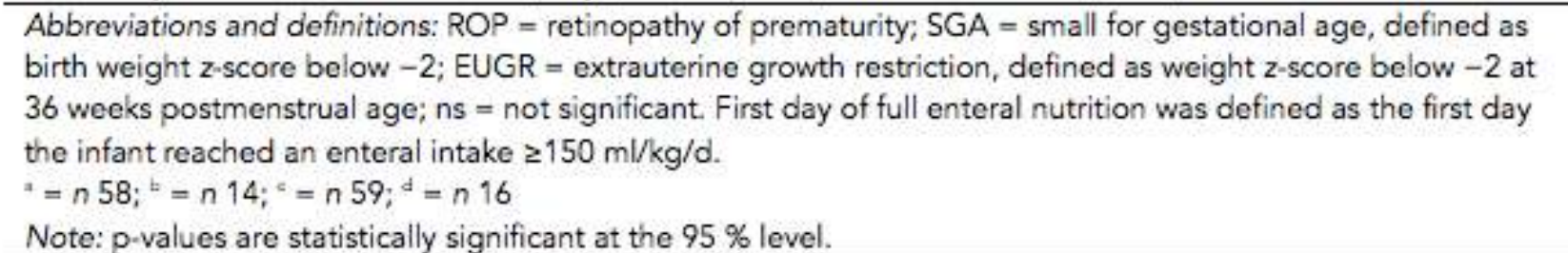 } \\
\hline
\end{tabular}

CONCLUSION

Higher early intake of enteral nutrition and improved postnatal growth is associated with normalized retinal angiogenesis, i.e. less retinopathy of prematurity, in extremely preterm infants.

Growth data is presented in Table 2 and Figure 1. Infants not developing ROP had higher postnatal growth z-scores throughout hospitalization compared to their ROP affected peers. Statistically significant differences between the two groups were primarily seen for z-score growth at actual time points, as opposed to delta growth, at postnatal age (PNA) 28 days, postmenstrual age (PMA) 32 and 36 weeks. After adjustment for gestational age and birth weight z-score in logistic regression models significance remained for $z$-score weight $(O R=0.175$, $p=0.030)$ and length $(O R=0.276, p=0.012)$ at PMA 32 weeks, and $z$-score length $(O R=0.412, p=0.043)$ at PMA 36 weeks.

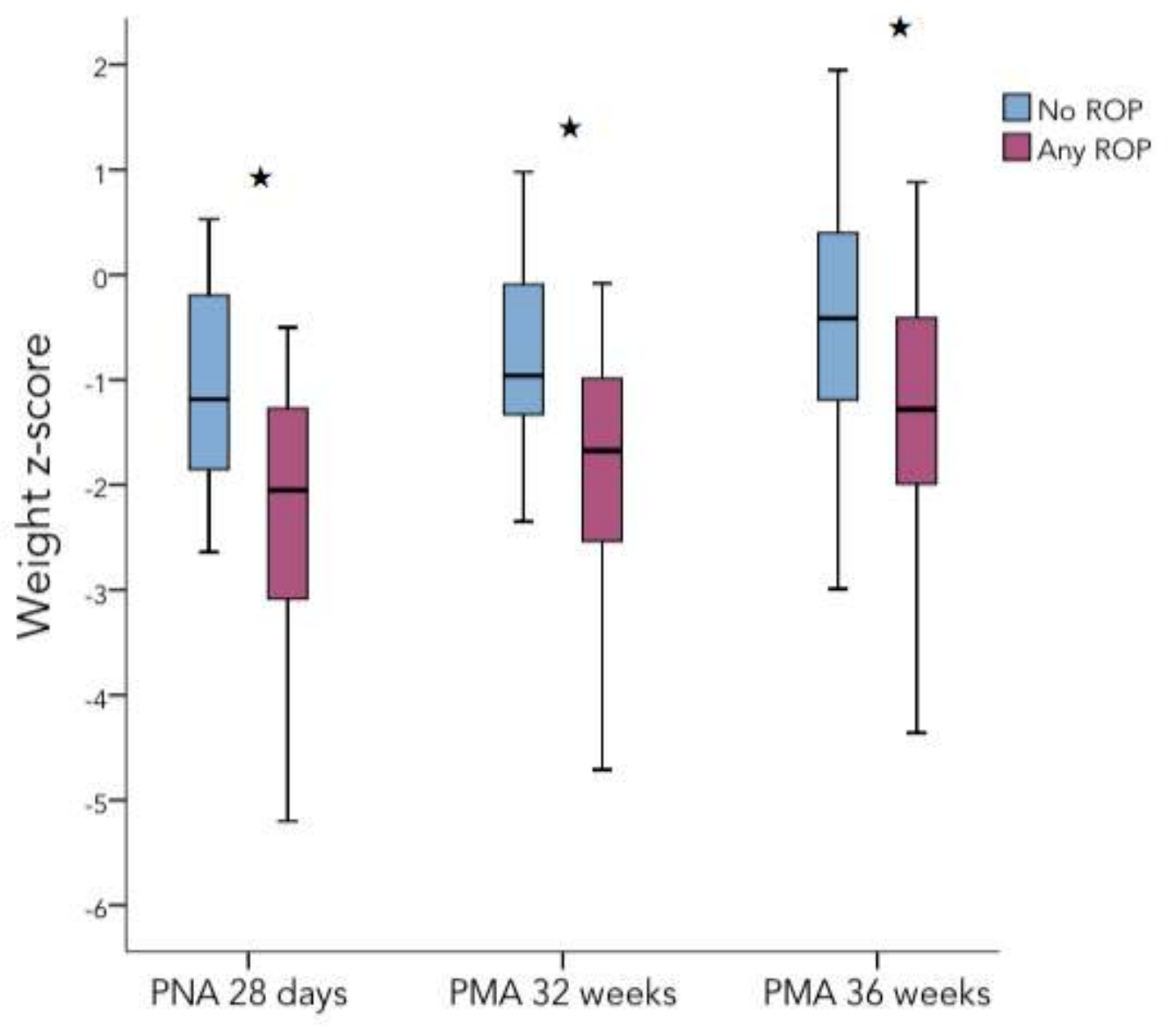

Figure 1. Difference in weight $z$-score at postnatal age 28 days and postmenstrual age 32 and 36 weeks, where infants with no ROP ( $n$ 17) had a better weight development than infants with any ROP ( $n 61)$. $\star p<0.05$.

Table 2. Postnatal growth and presence of ROP. Data is presented as median (min-max). Group any ROP included $n 61$ infants and group no ROP included $n 17$ infants.

\begin{tabular}{|c|c|c|c|c|c|c|c|c|c|}
\hline & \multicolumn{3}{|c|}{ POSTNATAL AGE 28 DAYS } & \multicolumn{3}{|c|}{ POSTMENSTRUAL AGE 32 WEEKS } & \multicolumn{3}{|c|}{ POSTMENSTRUAL AGE 36 WEEKS } \\
\hline & Any ROP & No ROP & $P$ value & Any ROP & No ROP & $P$ value & Any ROP & No ROP & P value \\
\hline$z$-score weight & $\begin{array}{l}-2.05 \\
(-5.20--0.50)^{a}\end{array}$ & $\begin{array}{l}-1.19 \\
(-2.64-0.53)^{b}\end{array}$ & 0.001 & $\begin{array}{l}-1.68 \\
(-4.90--0.08)^{a}\end{array}$ & $\begin{array}{l}-0.96 \\
(-2.35-0.98)\end{array}$ & 0.002 & $\begin{array}{l}-1.28 \\
(-5.86-0.88)^{c}\end{array}$ & $\begin{array}{l}-0.42 \\
(-2.99-1.95)^{d}\end{array}$ & 0.034 \\
\hline$z$-score length & $\begin{array}{l}-3.14 \\
(-6.16--0.47)\end{array}$ & $\begin{array}{l}-2.48 \\
(-3.44-0.35)^{f}\end{array}$ & 0.021 & $\begin{array}{l}-3.62 \\
(-6.30--0.95)^{9}\end{array}$ & $\begin{array}{l}-1.62 \\
(-2.96-0.38)\end{array}$ & $<0.001$ & $\begin{array}{l}-2.59 \\
(-8.09--0.53)^{h}\end{array}$ & $\begin{array}{l}-0.78 \\
(-2.67-1.53)^{d}\end{array}$ & $<0.001$ \\
\hline z-score $\mathrm{HC}$ & $\begin{array}{l}-1.98 \\
(-3.71--0.33)\end{array}$ & $\begin{array}{l}-1.60 \\
(-2.13--0.50)\end{array}$ & 0.049 & $\begin{array}{l}-1.88 \\
(-3.57-0.07)^{9}\end{array}$ & $\begin{array}{l}-0.95 \\
(-2.13-0.78)\end{array}$ & $<0.001$ & $\begin{array}{l}-1.45 \\
(-5.15-0.86)^{k}\end{array}$ & $\begin{array}{l}-0.61 \\
(-1.29-1.64)^{d}\end{array}$ & 0.001 \\
\hline z-score $\Delta$ weight & $\begin{array}{l}-1.20 \\
(-2.41-0.56)^{a}\end{array}$ & $\begin{array}{l}-1.15 \\
(-1.91--0.05)^{b}\end{array}$ & ns & $\begin{array}{l}-0.75 \\
(-2.71-0.82)^{a}\end{array}$ & $\begin{array}{l}-0.68 \\
(-1.62-0.40)\end{array}$ & ns & $\begin{array}{l}-0.05 \\
(-4.59-1.44)\end{array}$ & $\begin{array}{l}-0.61 \\
(-2.26-1.53)^{d}\end{array}$ & ns \\
\hline z-score $\Delta$ length & $\begin{array}{l}-2.06 \\
(-4.09-0.40)\end{array}$ & $\begin{array}{l}-2.04 \\
(-2.97--0.24)^{f}\end{array}$ & ns & $\begin{array}{l}-1.97 \\
(-5.05-0.78)^{m}\end{array}$ & $\begin{array}{l}-1.33 \\
(-2.70-0.45)\end{array}$ & 0.010 & $\begin{array}{l}-1.46 \\
(-5.01-1.30)^{n}\end{array}$ & $\begin{array}{l}-0.99 \\
(-1.64-1.36)^{d}\end{array}$ & ns \\
\hline z-score $\triangle \mathrm{HC}$ & $\begin{array}{l}-1.18 \\
(-2.79--0.08)^{\circ}\end{array}$ & $\begin{array}{l}-1.54 \\
(-2.49-0.23)\end{array}$ & ns & $\begin{array}{l}-1.29 \\
(-2.73-0.55)^{k}\end{array}$ & $\begin{array}{l}-1.09 \\
(-2.16-0.56)\end{array}$ & ns & $\begin{array}{l}-0.75 \\
(-4.11-2.47)^{m}\end{array}$ & $\begin{array}{l}-0.38 \\
(-1.41-1.75)^{d}\end{array}$ & ns \\
\hline
\end{tabular}

Abbreviations and definitions: ROP $=$ retinopathy of prematurity; $\Delta=$ delta, delta growth was defined as $z$-score at actual time point subtracted by $z$-score at birth; ns $=$ not significant. 